\title{
Functional Characterization of an Exopolysaccharide Produced by Bacillus sonorensis MJM60135 Isolated from Ganjang ${ }^{\text {S }}$
}

\author{
Sasikumar Arunachalam Palaniyandi ${ }^{1}$, Karthiyaini Damodharan ${ }^{2}$, Joo-Won Suh $^{2 *}$, and Seung Hwan Yang ${ }^{3 *}$ \\ ${ }^{1}$ Department of Biotechnology, Mepco Schlenk Engineering College, Mepco Nagar, Mepco Engineering College Post-626005, Sivakasi, \\ Tamilnadu, India \\ ${ }^{2}$ Center for Nutraceutical and Pharmaceutical Materials, College of Natural Science, Myongji University, Yongin 17058, Republic of Korea \\ ${ }^{3}$ Department of Biotechnology, Chonnam National University, Yeosu 59626, Republic of Korea
}

\author{
Received: December 16, 2017 \\ Revised: February 19, 2018 \\ Accepted: February 23, 2018 \\ First published online \\ March 15, 2018 \\ *Corresponding authors \\ J.W.S. \\ Phone: +82-31-330-6190; \\ Fax: +82-31-321-7361; \\ E-mail: jwsuh@mju.ac.kr \\ S.H.Y. \\ Phone: +82-61-659-7306; \\ Fax: +82-61-659-7309; \\ E-mail:ymichigan@jnu.ac.kr \\ S upplementary data for this \\ paper are available on-line only at \\ http://jmb.or.kr. \\ pISSN 1017-7825, eISSN 1738-8872 \\ Copyright $(2018$ by \\ The Korean Society for Microbiology \\ and Biotechnology
}

The present study focused on the production, characterization, and in vitro prebiotic evaluation of an exopolysaccharides (EPS) from Bacillus sonorensis MJM60135 isolated from ganjang (fermented soy sauce). Strain MJM60135 showed the highest production $(8.4 \pm 0.8 \mathrm{~g} / \mathrm{l})$ of EPSs compared with other isolates that were screened for EPS production based on ropy culture morphology. Furthermore, MJM60135 was cultured in $5 \mathrm{~L}$ of medium and the EPS was extracted by ethanol precipitation. The emulsification activity of the EPS was higher in toluene than in 0 -xylene. Fourier transform infrared spectroscopy analysis showed the presence of hydroxyl and carboxyl groups and glycosidic linkages. The isolated EPS contained mannose and glucose, as observed by thin-layer chromatography analysis of the EPS hydrolysate. Lactic acid bacteria (LAB) and pathogenic E. coli K99 and Salmonella enterica serovar Typhimurium were tested for their growth utilizing the EPS from B. sonorensis MJM60135 as the sole carbon source for its possible use as a prebiotic. All the tested LAB exhibited growth in the EPSsupplied medium compared with glucose as carbon source, whereas the pathogenic strains did not grow in the EPS-supplied medium. These findings indicate that the EPS from B. sonorensis MJM60135 has potential application in the bioremediation of hydrocarbons and could also be used as a prebiotic.

Keywords: Bacillus sonorensis, exopolysaccharide, prebiotic, lactic acid bacteria

\section{Introduction}

Bacterial exopolysaccharides (EPSs) are homo- or heteropolysaccharides secreted extracellularly by bacteria. EPSs play an important role in protecting the host cells against desiccation, antimicrobial compounds, bacteriophages, osmotic stress, and engulfment by protozoa, and aids in adherence to surfaces and biofilm formation [1]. Bacterial EPSs are structurally diverse and possess a variety of properties useful to human beings, which include high viscosity, stability over a wide range of $\mathrm{pH}$, temperature, and salt concentrations, non-ionic, non-toxic, gelling capacity, insolubility in most solvents, and high tensile strength [1]. These properties of EPSs make them applicable in the food, pharmaceutical, medicine, petrochemical, cosmetic, and other industries [1-4].

EPSs from several bacteria were reported to possess health-promoting effects, which include antitumor $[5,6]$, antiulcer [7], antiviral [8] and immunoregulatory [8], antiatherosclerotic [9], and prebiotic activities [3, 10, 11]. These health-promoting effects of EPSs make them valuable ingredients in the food industry.

Bacillus strains of food origin are regarded as safe and their EPSs can be used as prebiotics. Prebiotics are nondigestible polysaccharides, which act as substrates for fermentation by gut symbionts and stimulate the colonization of beneficial bacteria. EPSs from Bacillus strains have been reported to have antioxidant and free-radical scavenging activity [12, 13], hydroxyl radical scavenging and antityrosinase activities [14], anticytotoxic effect against Avarol 
[15], antitumor activity [6], anti-biofilm activity [16], immunological activity [17], and antiviral and immunoregulatory activities [8]. However, there are no reports on the prebiotic evaluation of EPSs from Bacillus strains.

This study focused on characterizing the properties of an EPS produced by Bacillus sonorensis strain MJM60135, such as emulsifying activity, carbohydrate composition, and the presence of functional groups by IR spectroscopy. Furthermore, the EPS was evaluated for its prebiotic potential by studying its effect as a carbon source for the growth of probiotic lactic acid bacteria (LAB) and enteric pathogens.

\section{Materials and Methods}

\section{Materials, Bacterial Strains, and Culture Conditions}

All the culture media used in this study were obtained from BD Difco (USA) unless otherwise stated. All analytical grade and spectroscopic grade chemicals used in this study were obtained from Sigma (USA). LAB such as Lactobacillus plantarum subsp. plantarum, L. plantarum, L. casei, L. brevis, L. fermentum, and Enterococcus durans were obtained from the Extract Collection of Useful Microorganisms (ECUM) library, Myongji University, South Korea. These strains were isolated from several fermented food sources, characterized, and maintained in the ECUM library. The LAB strains were routinely cultured in de Man, Rogosa, and Sharpe (MRS) agar medium at $37^{\circ} \mathrm{C}$. Pathogenic strains such as Escherichia coli K99 KCTC 2617 (E. coli K99) and Salmonella enterica subsp. enterica serovar. Typhimurium KCTC 2514 (Sal. Typhimurium) were obtained from Korean Collection for Type Cultures (KCTC), Republic of Korea. The pathogenic strains were routinely culture in Luria Bertani (LB) agar at $37^{\circ} \mathrm{C}$. Modified MRS (MMRS) medium ( $10 \mathrm{~g} / 1$ casamino acids, $2 \mathrm{~g} / 1 \mathrm{Na}_{2} \mathrm{HPO}_{4}, 5 \mathrm{~g} / 1$ sodium acetate, $2 \mathrm{~g} / 1$ triammonium citrate, $0.2 \mathrm{~g} / 1 \mathrm{MgSO}_{4} \bullet 7 \mathrm{H}_{2} \mathrm{O}, 0.2 \mathrm{~g} / 1$ $\mathrm{MnSO}_{4} \bullet 4 \mathrm{H}_{2} \mathrm{O}$, and $1 \mathrm{~g} / 1$ Tween 80 ) was used for the study of EPS utilization by LAB strains. M9 minimal salts [18] containing $1 \%$ casamino acids (M9 medium) was used for the EPS utilization study of pathogenic strains.

\section{Screening of EPS-Producing Strains and Culture Conditions}

Ganjang (fermented soy sauce) samples (obtained from a local market) were serially diluted and the suspension was plated on tryptic soy agar (TSA) and incubated for 2 days at $37^{\circ} \mathrm{C}$. Colonies showing distinct morphology were picked and subcultured on TSA. A total of 37 isolates were picked and subcultured on TSA. The isolates were visibly screened for slimy appearance and ropiness of the culture. Nine isolates that showed a ropy appearance were used for further study.

\section{Extraction and Quantification of Exopolysaccharides from Bacillus Strains}

The selected Bacillus strains were cultured in tryptic soy broth
(TSB) and agitated at $37^{\circ} \mathrm{C}$ for $48 \mathrm{~h}$. The culture was centrifuged at $5,000 \times g$ for $10 \mathrm{~min}$ to remove cells. The supernatant was boiled for $10 \mathrm{~min}$ to inactivate enzymes. The supernatant was decolorized with activated carbon in a water bath at $40^{\circ} \mathrm{C}$ for $30 \mathrm{~min}$ [6] and then deproteinized as described previously [19]. The deproteinized supernatant was subsequently centrifuged at 5,000 $\times g$ for $10 \mathrm{~min}$, mixed with 4 volumes of $95 \%$ ethanol, and kept overnight at $4^{\circ} \mathrm{C}$. The mixture was then centrifuged at $8,000 \times g$ for $10 \mathrm{~min}$, and the pellet was collected and dissolved in sterile distilled water. The solution was centrifuged at 3,000 $\times g$ for $10 \mathrm{~min}$, and the supernatant was dialyzed against sterile distilled water for $24 \mathrm{~h}$. The EPS solution was freeze dried and stored at $4^{\circ} \mathrm{C}$ until used. The EPS content was measured by the phenol-sulfuric acid method [20] using glucose as a standard.

\section{Identification of MJM60315}

The genomic DNA of the strains were isolated using a genomic DNA isolation kit (GeneALL, Korea), following the manufacturer's instruction. The 16S rRNA gene sequence of the strains was amplified using 27F (5'-AGAGTTTGATCC TGGCTCAG-3') and 1492R (5'-GGTTACCTTGTTACGACTT-3') primers and the PCR products were sequenced by SolGent, a sequencing company (SolGent, Republic of Korea), with the primers 27F and 785F (5'GGATTAGATACCCTGGTA-3') to get a near full length of the $16 \mathrm{~S}$ rRNA gene sequence. The sequences were compared with those in the EzBioCloud database [21] using the BLAST program and a neighbor-joining phylogenetic tree was constructed using MEGA 6 software [22].

\section{Emulsifying Activity of the EPS from MJM60315}

Emulsification assays were carried out according to Fusconi et al. [23]. B. sonorensis MJM60315 was cultured in TSB for $24 \mathrm{~h}$. The supernatant was collected by centrifugation and $3 \mathrm{ml}$ of the supernatant was mixed with an equal volume of hydrocarbon compound (toluene or $o$-xylene) in a test tube. The tubes were vortexed for $2 \mathrm{~min}$ and incubated at $25^{\circ} \mathrm{C}$ for $24 \mathrm{~h}$. The emulsifying activity was investigated after $24 \mathrm{~h}$ and the emulsification index $\left(E_{24}\right)$ was calculated as follows: $E_{24}=$ (height of emulsion layer/ total height $) \times 100$. A higher emulsification index indicates higher emulsification activity [23]. Triton X-100 (10\% in TSB) was used as a positive control for comparison. A blank was prepared with distilled water without EPS and mixed with hydrocarbon compounds.

\section{Fourier Transform Infrared Spectra of the EPS}

Functional groups present in the purified EPS were determined by Fourier transform infrared (FTIR) spectroscopy. Samples for infrared analysis were prepared by mixing with spectroscopygrade $\mathrm{KBr}$ and prepared in the form of pellets at a pressure of $1 \mathrm{MPa}$. The pellets were of about $10 \mathrm{~mm}$ diameter and $1 \mathrm{~mm}$ thickness. The FTIR spectral data were recorded with a Varian 2000 FTIR spectrometer (Varian, Inc., USA). FTIR spectra were recorded covering the $4,000-400 \mathrm{~cm}^{-1}$ region. 


\section{EPS Composition Analysis}

The lyophilized EPS $(10 \mathrm{mg})$ was hydrolyzed with $6 \mathrm{M}$ trifluoroacetic acid (TFA) for $6 \mathrm{~h}$ at $100^{\circ} \mathrm{C}$. The TFA was removed using a rotary vacuum evaporator, the hydrolyzed solution was neutralized with $15 \mathrm{M}$ ammonia solution $(0.32 \mathrm{ml})$ [13], and the resultant hydrolysate was analyzed by thin-layer chromatography (TLC) to determine the monosaccharide composition. The monosaccharide standard was also treated with $6 \mathrm{M}$ TFA and neutralized with ammonia as described above. The EPS hydrolysates were separated using Silica Gel 60 TLC using pre-coated plates (Merck, Germany) developed with a mobile phase of ethylacetate: n-propanol: acetic acid: water $(4: 2: 2: 1(\mathrm{v} / \mathrm{v} / \mathrm{v} / \mathrm{v}))$. The separated sugars were visualized with an orcinol-sulfuric acid spray reagent.

Effect of EPSs on Growth of Lactic Acid Bacteria and Enteric Pathogens

The growth and EPS utilization by LAB such as L. plantarum subsp. plantarum, L. plantarum, L. casei, L. brevis, L. fermentum, and Enterococcus durans were evaluated in MMRS medium with $1 \%$ EPS at $37^{\circ} \mathrm{C}$ for $12 \mathrm{~h}$. LAB growth was also analyzed in MMRS with $1 \%$ glucose (positive control). The growth of the pathogenic bacteria E. coli $\mathrm{K} 99$ and S. Typhimurium was monitored using M9 medium supplemented with $1 \%$ EPS and $1 \%$ glucose (positive control) as the carbon source separately. The growth study was done using 96-microwell plates and monitored every $1 \mathrm{~h}$ in an automated microtiter plate (Infinite M200 PRO; Tecan, Austria).

\section{Statistical Analyses}

Experiments were repeated at least three times. The data are expressed as the mean \pm standard deviation. Data for the emulsification index were analyzed by one-way anova and means were compared by the Tukey's test using SPSS statistics (IBM, USA).

\section{Results}

\section{Screening of EPS-Producing Microbes}

EPS-producing isolates were selected on the basis of a ropy or slimy appearance of the colony. Colonies showing a slimy appearance were picked and subcultured in TSA and incubated for 2 days at $37^{\circ} \mathrm{C}$. Totally nine isolates were collected from the ganjang samples. The EPS-producing isolates were named as EPS-producing bacteria 1 to 9, abbreviated as EPSB1 to 9. The morphology of the isolates is shown in Fig. S1.

\section{Quantification of the EPS}

The EPS produced by Bacillus isolates was quantified by the phenol-sulfuric acid method. The results of the analysis showed that EPSB6 produced the highest amount $(8.4 \pm$ $0.8 \mathrm{~g} / \mathrm{l})$ of EPS, followed by EPSB9 with $7.0 \pm 0.76 \mathrm{~g} / \mathrm{l}$, EPSB5 with $5.6 \pm 0.4 \mathrm{~g} / 1$, and EPSB1 with $4.0 \pm 1.1 \mathrm{~g} / 1$ (Fig. 1). The

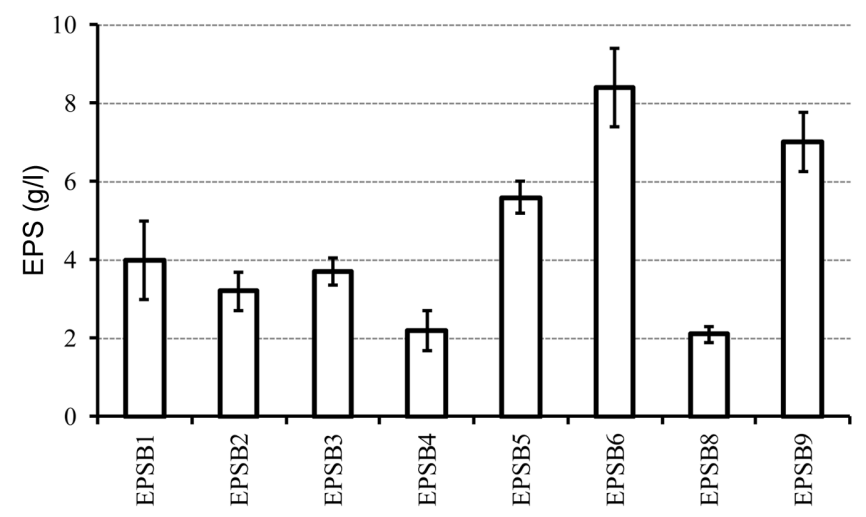

Fig. 1. Production of exopolysaccharides in liquid medium by EPS-producing Bacillus isolates from ganjang.

EPSB6 was renamed as MJM60315 after initial screening.

lowest amount of EPS $(2.3 \pm 0.34 \mathrm{~g} / \mathrm{l})$ was produced by EPSB8. The highest EPS-producing isolate EPSB6 was deposited in the ECUM library at Myongji University under the strain number MJM60315. This strain will be referred to as MJM60315 hereafter. The culture morphology and the EPS productivity of strain MJM60315 are shown in Fig. 2.

\section{Identification of MJM60315}

The 16S rRNA gene sequence was 1,475 bp for MJM60315. BLAST search of the EzBioCloud database showed that the $16 \mathrm{~S}$ rRNA gene sequence of MJM60315 showed $\geq 99 \%$ similarity with B. sonorensis NBRC $101234^{\mathrm{T}}$. Fig. 3 shows the phylogenetic relationship of the isolates to known species of the genus Bacillus.

\section{Emulsifying Activity of the EPS from MJM60315}

The emulsification index $\left(\mathrm{E}_{24}\right)$ of the EPS from MJM60315 was higher in toluene ( $\geq 60 \%$ ) than 0 -xylene (Fig. 4).

A

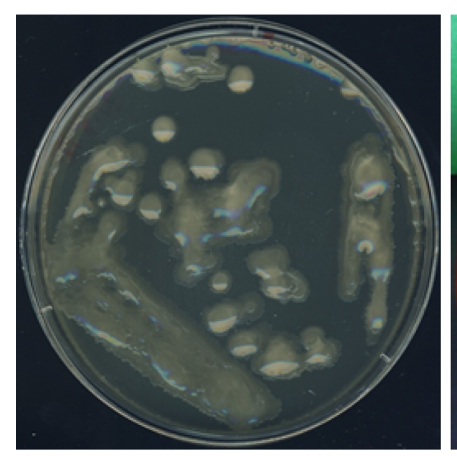

B

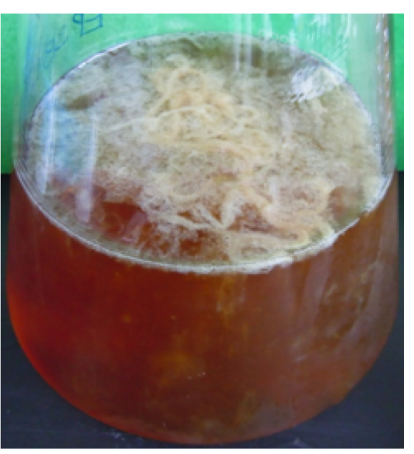

Fig. 2. Morphology of Bacillus sonorensis MJM60315 cultured on tryptic soy agar (A) and its production of exopolysaccharides in tryptic soy broth $(\mathbf{B})$. 


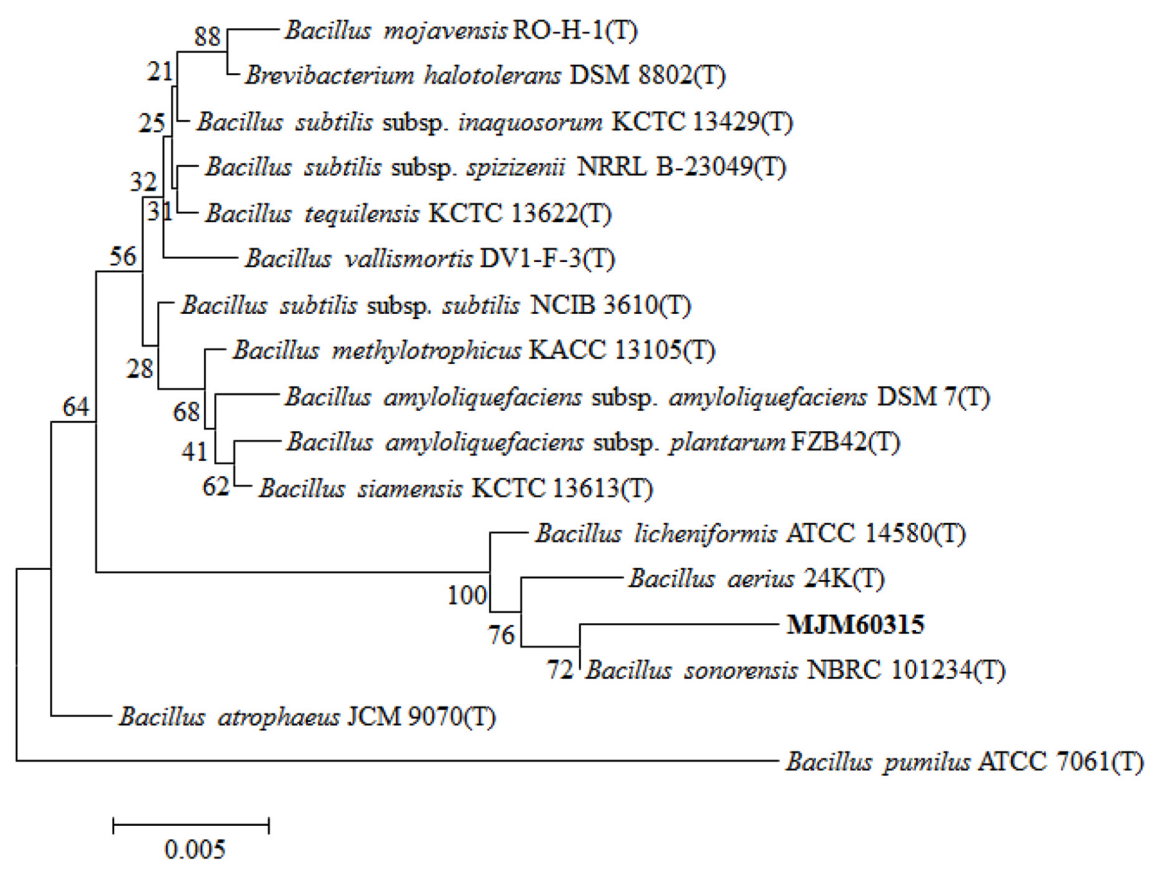

Fig. 3. Identification of exopolysaccharide (EPS)-producing strains.

Phylogenetic analysis of EPS-producing strain MJM60315 from ganjang, based on 16S rRNA gene sequencing.

\section{FTIR Spectral Analysis of the EPS from MJM60315}

The FTIR spectrum of the EPS from MJM60315 showing the functional groups in the $4,000-400 \mathrm{~cm}^{-1}$ region is given in Fig. 5. In the IR spectrum, the peak at $3,420 \mathrm{~cm}^{-1}$ corresponds to the $\mathrm{OH}$ stretch and the peak at 2,920 corresponds to the $\mathrm{CH}$ stretch. The broad stretch of $\mathrm{C}-\mathrm{O}-\mathrm{C}$, C-O at between 1,000 and $1,200 \mathrm{~cm}^{-1}$ corresponds to carbohydrates [24]. A peak at 1,366 $\mathrm{cm}^{-1}$ could be assigned

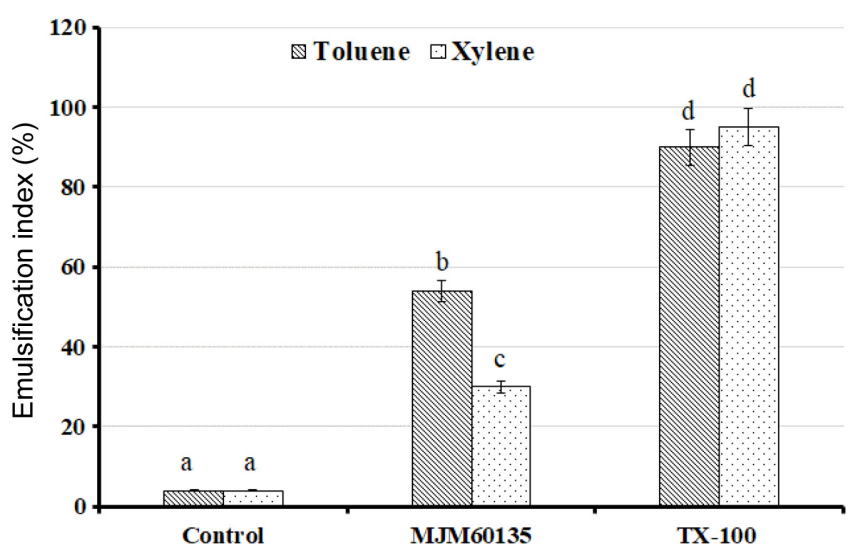

Fig. 4. Emulsification activity of the exopolysaccharide from Bacillus sonorensis MJM60315, tested in toluene and xylene. Bars with different letters indicate they are significantly different $(p<0.05)$ from each other. to the $\mathrm{C}=\mathrm{O}$ stretch of the $\mathrm{COO}-$ and $\mathrm{C}-\mathrm{O}$ bond from $\mathrm{COO}-$ [24]. The band at $1,162 \mathrm{~cm}^{-1}$ corresponds to a glycosidic linkage, as 1,4-glycosidic linkage in polysaccharides gives absorption bands in the range of $1,175-1,140 \mathrm{~cm}^{-1}$ [25] (Fig. 5).

\section{Monosaccharide Composition of the EPS from MJM60315}

Hydrolysis of the EPS with $6 \mathrm{M}$ TFA and subsequent analysis of the EPS hydrolysate by TLC showed the

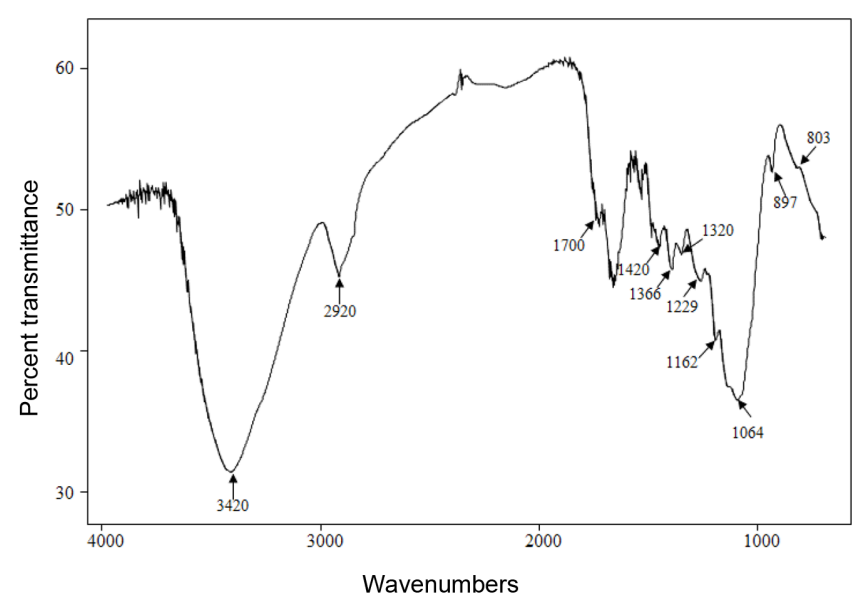

Fig. 5. FTIR analysis of the exopolysaccharide preparation from MJM60315. 


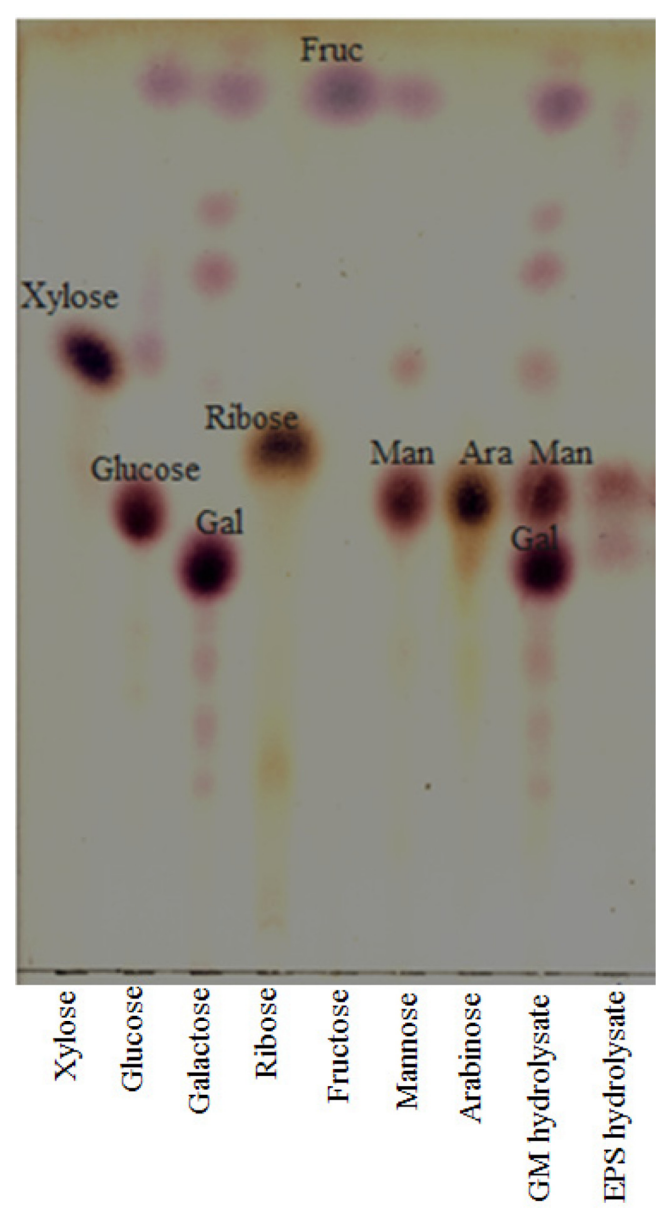

Fig. 6. TLC analysis of monosaccharides from the MJM60135 exopolysaccharide hydrolysate.

GM, Galactomannan.

presence of mannose and glucose as principal components of the EPS from MJM60315 (Fig. 6).

\section{Effect of the EPS on the Growth of Lactic Acid Bacteria and Enteric Pathogens}

Of the various LAB strains tested, Enterococcus durans showed the lowest growth rate in EPS-supplied medium (Fig. 7B) compared with growth in glucose-supplied medium (Fig. 7A). Maximal growth in EPS-supplied medium was observed for the L. plantarum strains, L. brevis, and L. fermentum. L.casei showed moderate growth in EPSsupplied medium. Pathogenic strains E.coli K99 and $S$. Typhimurium did not show any growth in EPS-supplied medium (Fig. 7D) compared with glucose-supplied medium (Fig. 7C), indicating that these strains could not utilize EPS as a carbon source.

\section{Discussion}

Screening of strains showing a ropy culture morphology from Korean ganjang (fermented soy sauce) resulted in the isolation of a B. sonorensis strain, MJM60315. The occurrence of $B$. sonorensis in soy-based fermented foods was previously reported in Chinese soy sauce [26] and in Indian kinema, a fermented soy-based food [27]. The MJM60315 strain produced $>8 \mathrm{~g} / 1$ of EPSs, which was lower than the level of EPSs production reported in B. polymyxa KCTC 8648P [28], B. licheniformis 8-37-0-1 [17], and B. licheniformis KS-17 [14]. However, MJM60315 EPS production was higher than that of several previously reported Bacillus strains, such as seaweed-associated B. licheniformis [29], B. licheniformis LMG 19409 [30], and B. thermoantarcticus [31].

The EPS from B. sonorensis MJM60315 acts as biosurfactant and showed emulsifying property with aromatic hydrocarbons such as toluene and $o$-xylene. These solvents were indicated as among the main groundwater-contaminant and healthrisk group [23]. Owing to its emulsifying activity, MJM60315 EPS could find potential applications in hydrocarbon bioremediation. Biosurfactants are of two types: lowmolecular-weight biosurfactants such as glycolipids, and high-molecular-weight extracellular polymers such as polysaccharides, proteins, lipopolysaccharides, lipoproteins, or complex mixtures of these biopolymers [32]. The EPS from B. sonorensis MJM60315 could be classified as a highmolecular-weight biosurfactant.

The FTIR spectrum of the EPS showed the presence of carboxyl and hydroxyl groups, which are important for binding divalent cations and for the flocculation process [30]. The EPS produced by B. sonorensis MJM60315 was observed to be a heteropolysaccharide-containing mannose and glucose based on TLC analysis of the EPS hydrolysate. This result was similar to that of previous reports on EPSs produced by B. amyloliquefaciens LPL061 [33], B. thermoantarcticus isolated close to the crater of Mount Melbourne in Antarctica [31], Bacillus isolated from a shallow hydrothermal vent at Vulcano island [34], halophilic thermotolerant Bacillus isolated from a marine hot spring at Vulcano Island [35], and a B. licheniformis strain isolated from ropy cider [30] containing mannose and glucose as the main components. Although all of these strains were isolated from various locations and in different ecological niches, they appear to produce EPSs containing similar composition.

The EPS from B. sonorensis MJM60315 showed potential prebiotic property by selectively supporting the growth of 

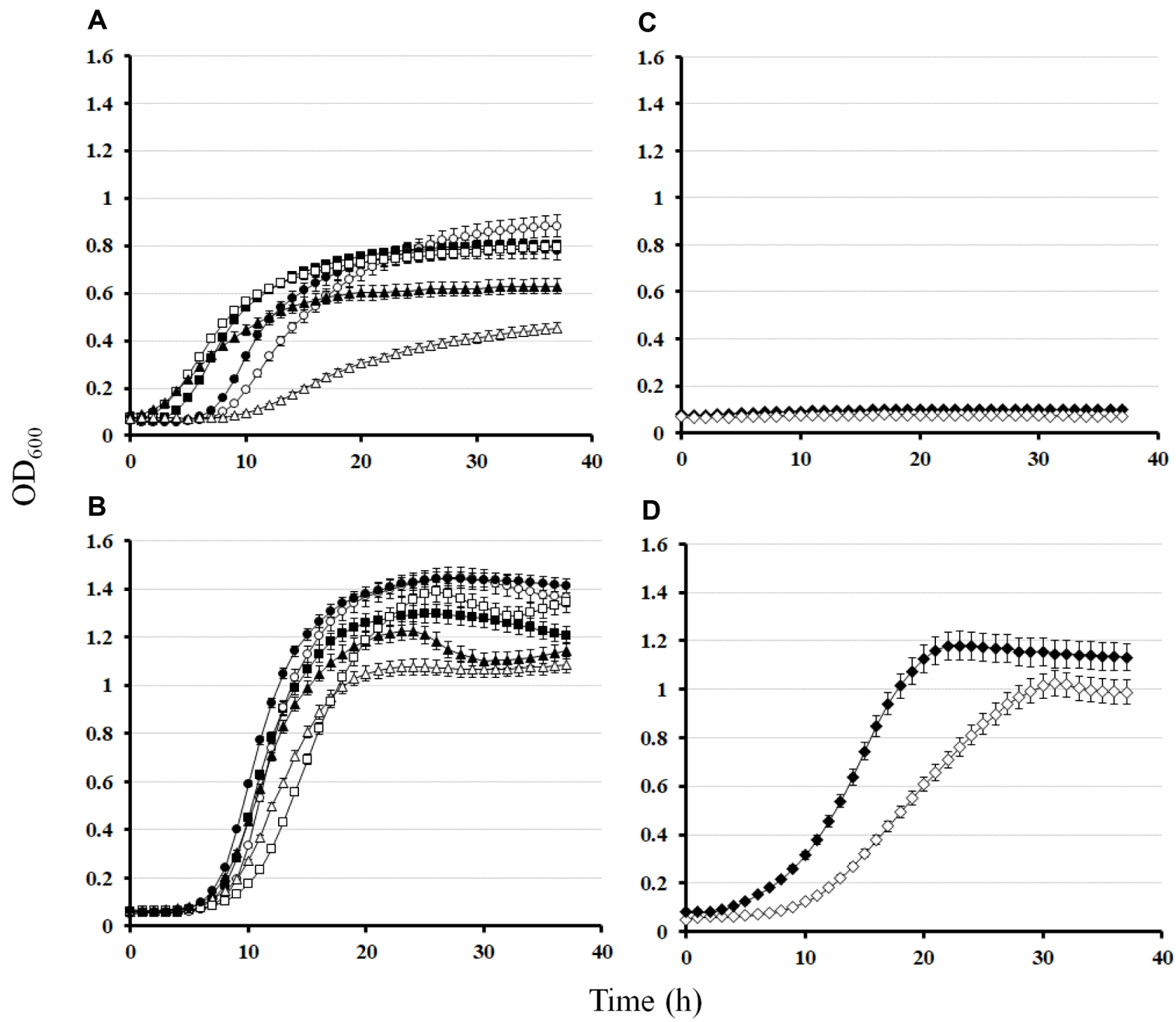

Fig. 7. Growth of LAB and pathogenic strains in MJM60315 exopolysaccharide (EPS) (1\%)-supplied medium as the carbon source compared with that in glucose (1\%)-supplied medium.

(A) MMRS-EPS; (B) MMRS-Glucose; (C) M9 medium-EPS; (D) M9 medium-Glucose. (@) Lactobacillus plantarum subsp. plantarum; ( $($ ) L. plantarum; $(\boldsymbol{\square})$ L. brevis; $(\square)$ L. fermentum; $(\Delta)$ L. casei; $(\triangle)$ Enterococcus durans; $(\diamond)$ E. coli K99; $(\diamond)$ Salmonella Typhimurium.

LAB. The pathogenic strains were unable to grow in the medium containing EPS as a carbon source, indicating that in the presence of EPS, LAB will be selectively enriched, whereas pathogenic Enterobacteriaceae members will be suppressed. A similar result was observed in the cases of EPS from Weissella cibaria A2, W. confusa A9, L. plantarum A3, and Pediococcus pentosaceus 5S4, which selectively enhanced the growth of Bifidobacterium and Lactobacillus / Enterococcus groups whereas Clostridia were suppressed [11]. In another study, EPS produced by Lactococcus lactis 1.8 supported the growth of Bifidobacterium angulatum LMG 11568, Bifidobacterium breve LMG 11084, Bifidobacterium dentium LMG 10507, and Bifidobacterium pseudocatenulatum
LMG 10505T; however, Clostridium perfringens LMG 11264T and C. clostridioforme DSM 933T were unable to utilize the EPS [3].

In conclusion, B. sonorensis MJM60315 produced a watersoluble heteropolysaccharide, which is composed of mannose and glucose and exhibited emulsifying property with aromatic hydrocarbons such as toluene and 0 -xylene. The FTIR spectrum of the EPS produced by $B$. sonorensis MJM60315 showed chemical groups important for flocculation activity. Additionally, growth study using EPS as the sole carbon source showed that it could specifically support the growth of LAB while suppressing the growth of E. coli and S. Typhimurium. These results indicate that the 
EPS from B. sonorensis MJM60315 has potential application in the bioremediation of hydrocarbon contaminants and as a prebiotic in the food industry.

\section{Acknowledgments}

This research was supported by the Basic Science Research Program through the National Research Foundation of Korea (NRF) funded by the Ministry of Education (NRF2017R1D1A3B03027816).

\section{Conflict of Interest}

The authors have no financial conflicts of interest to declare.

\section{References}

1. Nwodo UU, Green E, Okoh AI. 2012. Bacterial exopolysaccharides: functionality and prospects. Int. J. Mol. Sci. 13: 14002-14015.

2. Prajapat J, Patel A. 2013. Food and health applications of exopolysaccharides produced by lactic acid bacteria. Adv. Dairy Res. 1: 1-8.

3. Grosu-Tudor S-S, Zamfir M, Meullen RVD, Falony G, Vuyst LD. 2013. Prebiotic potential of some exopolysaccharides produced by lactic acid bacteria. Rom. Biotechnol. Lett. 18: 8666-8676.

4. De Vuyst L, Degeest B. 1999. Heteropolysaccharides from lactic acid bacteria. FEMS Microbiol. Rev. 23: 153-177.

5. Liu J, Luo J, Ye H, Zeng X. 2012. Preparation, antioxidant and antitumor activities in vitro of different derivatives of levan from endophytic bacterium Paenibacillus polymyxa EJS-3. Food Chem. Toxicol. 50: 767-772.

6. Chen Y-T, Yuan Q, Shan L-T, Lin M-A, Cheng D-Q, Li C-Y. 2013. Antitumor activity of bacterial exopolysaccharides from the endophyte Bacillus amyloliquefaciens sp. isolated from Ophiopogon japonicus. Oncol. Lett. 5: 1787-1792.

7. Rasulov MM, Kuznetsov IG, Slutskii LI, Velikaia MV, Zabozlaev AG, Voronkov MG. 1993. The ulcerostatic effect of the exopolysaccharide from Bacillus mucilaginosus and its possible mechanisms. Biull. Eksp. Biol. Med. 116: 504-505.

8. Arena A, Maugeri TL, Pavone B, Iannello D, Gugliandolo C, Bisignano G. 2006. Antiviral and immunoregulatory effect of a novel exopolysaccharide from a marine thermotolerant Bacillus licheniformis. Int. Immunopharmacol. 6: 8-13.

9. Uchida M, Ishii I, Inoue C, Akisato $Y$, Watanabe $K$, Hosoyama S, et al. 2010. Kefiran reduces atherosclerosis in rabbits fed a high cholesterol diet. J. Atheroscler. Thromb. 17: 980-988.

10. Bello FD, Walter J, Hertel C, Hammes WP. 2001. In vitro study of prebiotic properties of levan-type exopolysaccharides from lactobacilli and non-digestible carbohydrates using denaturing gradient gel electrophoresis. Syst. Appl. Microbiol. 24: $232-237$

11. Hongpattarakere T, Cherntong N, Wichienchot S, Kolida S, Rastall RA. 2012. In vitro prebiotic evaluation of exopolysaccharides produced by marine isolated lactic acid bacteria. Carbohydr. Polym. 87: 846-852.

12. Kodali VP, Sen R. 2008. Antioxidant and free radical scavenging activities of an exopolysaccharide from a probiotic bacterium. Biotechnol. J. 3: 245-251.

13. Kodali VP, Perali RS, Sen R. 2011. Purification and partial elucidation of the structure of an antioxidant carbohydrate biopolymer from the probiotic bacterium Bacillus coagulans RK-02. J. Nat. Prod. 74: 1692-1697.

14. Song Y-R, Jeong D-Y, Baik S-H. 2013. Optimal production of exopolysaccharide by Bacillus licheniformis KS-17 isolated from kimchi. Food Sci. Biotechnol. 22: 417-423.

15. Spano A, Gugliandolo C, Lentini V, Maugeri TL, Anzelmo G, Poli A, et al. 2013. A novel EPS-producing strain of Bacillus licheniformis isolated from a shallow vent off Panarea island (Italy). Curr. Microbiol. 67: 21-29.

16. Sayem SM, Manzo E, Ciavatta L, Tramice A, Cordone A, Zanfardino A, et al. 2011. Anti-biofilm activity of an exopolysaccharide from a sponge-associated strain of Bacillus licheniformis. Microb. Cell Fact. 10: 74.

17. Liu C, Lu J, Lu L, Liu Y, Wang F, Xiao M. 2010. Isolation, structural characterization and immunological activity of an exopolysaccharide produced by Bacillus licheniformis 8-37-0-1. Bioresour. Technol. 101: 5528-5533.

18. Bren A, Park JO, Towbin BD, Dekel E, Rabinowitz JD, Alon U. 2016. Glucose becomes one of the worst carbon sources for E. coli on poor nitrogen sources due to suboptimal levels of cAMP. Sci. Rep. 6: 24834.

19. Wang X, Yuan Y, Wang K, Zhang D, Yang Z, Xu P. 2007. Deproteinization of gellan gum produced by Sphingomonas paucimobilis ATCC 31461. J. Biotechnol. 128: 403-407.

20. Masuko T, Minami A, Iwasaki N, Majima T, Nishimura S, Lee YC. 2005. Carbohydrate analysis by a phenol-sulfuric acid method in microplate format. Anal. Biochem. 339: 69-72.

21. Yoon SH, Ha SM, Kwon S, Lim J, Kim Y, Seo H, et al. 2017. Introducing EzBioCloud: a taxonomically united database of 16S rRNA gene sequences and whole-genome assemblies. Int. J. Syst. Evol. Microbiol. 67: 1613-1617.

22. Tamura K, Stecher G, Peterson D, Filipski A, Kumar S. 2013. MEGA6: molecular evolutionary genetics analysis version 6.0. Mol. Biol. Evol. 30: 2725-2729.

23. Fusconi R, Nascimento Assunção RM, de Moura Guimarães R, Rodrigues Filho G, Eduardo da Hora Machado A. 2010. Exopolysaccharide produced by Gordonia polyisoprenivorans CCT 7137 in GYM commercial medium and sugarcane molasses alternative medium: FT-IR study and emulsifying activity. Carbohydr. Polym. 79: 403-408. 
24. Wang Y, Ahmed Z, Feng W, Li C, Song S. 2008. Physicochemical properties of exopolysaccharide produced by Lactobacillus kefiranofaciens ZW3 isolated from Tibet kefir. Int. J. Biol. Macromol. 43: 283-288.

25. Nikonenko NA, Buslov DK, Sushko NI, Zhbankov RG. 2000. Investigation of stretching vibrations of glycosidic linkages in disaccharides and polysaccharides with use of IR spectra deconvolution. Biopolymers 57: 257-262.

26. Yin WF, Tung HJ, Sam CK, Koh CL, Chan KG. 2012. Quorum quenching Bacillus sonorensis isolated from soya sauce fermentation brine. Sensors 12: 4065-4073.

27. Chettri R, Bhutia MO, Tamang JP. 2016. Poly-gammaglutamic acid (PGA)-producing Bacillus species isolated from Kinema, Indian fermented soybean food. Front. Microbiol. 7: 971.

28. Lee IY, Seo WT, Kim GJ, Kim MK, Ahn SG, Kwon GS, et al. 1997. Optimization of fermentation conditions for production of exopolysaccharide by Bacillus polymyxa. Bioprocess Eng. 16: 71-75.

29. Singh RP, Shukla MK, Mishra A, Kumari P, Reddy CRK, Jha B. 2011. Isolation and characterization of exopolysaccharides from seaweed associated bacteria Bacillus licheniformis.
Carbohydr. Polym. 84: 1019-1026.

30. Larpin S, Sauvageot N, Pichereau V, Laplace JM, Auffray Y. 2002. Biosynthesis of exopolysaccharide by a Bacillus licheniformis strain isolated from ropy cider. Int. J. Food Microbiol. 77: 1-9.

31. Manca MC, Lama L, Improta R, Esposito E, Gambacorta A, Nicolaus B. 1996. Chemical composition of two exopolysaccharides from Bacillus thermoantarcticus. Appl. Environ. Microbiol. 62: 3265-3269.

32. Ron EZ, Rosenberg E. 2001. Natural roles of biosurfactants. Environ. Microbiol. 3: 229-236.

33. Han Y, Liu E, Liu L, Zhang B, Wang Y, Gui M, et al. 2015. Rheological, emulsifying and thermostability properties of two exopolysaccharides produced by Bacillus amyloliquefaciens LPL061. Carbohydr. Polym. 115: 230-237.

34. Nicolaus B, Panico A, Manca MC, Lama L, Gambacorta A, Maugeri T, et al. 2000. A thermophilic Bacillus isolated from an Eolian shallow hydrothermal vent able to produce exopolysaccharides. Syst. Appl. Microbiol. 23: 426-432.

35. Maugeri TL, Gugliandolo C, Caccamo D, Panico A, Lama L, Gambacorta A, et al. 2002. A halophilic thermotolerant Bacillus isolated from a marine hot spring able to produce a new exopolysaccharide. Biotechnol. Lett. 24: 515-519. 